\title{
Quality Improvement Learning Collaborative Improves Timely Newborn Follow-Up Appointments
}

Emily Scott MD ${ }^{1}$, Stephen Downs MD ${ }^{1}$, Amy Pottenger BA, CCRP and Michele Saysana $\mathrm{MD}^{1}$

1. Department of Pediatrics, Indiana University School of Medicine

\begin{abstract}
BACKGROUND

American Academy of Pediatrics guidelines indicate that newborns should follow up with their primary care providers within three days of discharge from the newborn nursery. Many barriers exist to achieving timely follow-up, with potential implications on a newborn's health. The goal of this project was to improve rates of timely newborn followup through a nine-month quality improvement learning collaborative (QILC). Timely newborn follow-up was defined as an appointment scheduled within three days of newborn discharge.
\end{abstract}

\section{METHODS}

Both inpatient hospitalist and outpatient pediatric practices were eligible to participate. Inpatient and outpatient practices aimed to have $75 \%$ of newborns scheduled appropriately by six months into the project. In addition, outpatient practices aimed to have $60 \%$ of newborns seen appropriately by their provider. All practices aimed to have their progress sustained at conclusion of the QILC. Practices submitted data at baseline and nine subsequent phases. Monthly webinars featured a quality improvement didactic, data review, and discussion of practices' changes, successes, and challenges.

\section{RESULTS}

Eleven practices and 24 physicians participated in the QILC. Aggregate data from the practices showed continual improvement in all measured newborn scheduling metrics throughout the nine-month learning collaborative, with sustainment of progress over the last three months of the QILC.

\section{CONCLUSION}

A QILC is successful for increasing timely newborn follow-up for both the newborn hospitalist and outpatient pediatrician. Pediatric providers can learn from others' strategies and successes to incorporate meaningful changes in their practice.

This is the author's manuscript of the article published in final edited form as:

Scott, E., Downs, S., Pottenger, A., \& Saysana, M. (2019). Quality Improvement Learning Collaborative Improves Timely Newborn Follow-Up Appointments. Joint Commission Journal on Quality and Patient Safety, 45(12), 808-813. https://doi.org/10.1016/j.jcjq.2019.08.005 
Timely newborn follow-up is essential to ensure that newborns complete a safe transition into their medical home. When newborn follow-up is scheduled beyond the first days after discharge, it can delay the diagnosis of serious medical problems such as jaundice, breastfeeding failure, and late-presenting congenital defects. Early readmission of previously well newborns is most commonly due to jaundice and feeding problems, both of which are frequently preventable. ${ }^{1}$ When newborn follow-up visits are delayed, pediatricians also miss a crucial opportunity to provide important anticipatory guidance in areas such as breastfeeding, safe sleep, and tobacco cessation.

The American Academy of Pediatrics (AAP) Bright Futures Guidelines recommend that all newborns discharged from the well newborn nursery at less than 96 hours of life have a follow-up appointment with their primary care provider within two to three days of hospital discharge. ${ }^{2}$ The AAP has also endorsed follow-up within 48 hours of hospital discharge through other practice guidelines for breastfeeding newborns, newborns at risk for jaundice, and newborns discharged from the hospital at less than 48 hours of life. 345 Despite these recommendations, newborns frequently are not evaluated by a pediatric provider within the first three days after hospital discharge.

Delayed newborn outpatient follow-up has been discussed in the literature. A recent study from Utah reported that only $15 \%$ of newborns had follow-up within the recommended time frame. In that study, newborns with appropriate, timely follow-up had a decreased rate of hospital readmission in the first month of life. ${ }^{6}$ This mirrors previously published data from Ohio that early follow-up visits are associated with a significantly decreased risk of hospital readmission. ${ }^{7}$ A study from a large academic hospital in New York reported that $44 \%$ of their newborns did not have follow-up scheduled within three days of hospital discharge. In that study, a longer interval to the follow-up appointment was associated with Medicaid insurance, older gestational age, cesarean section delivery, and discharge home on a Thursday or Friday. ${ }^{8}$ However, this same group found that timing of the newborn follow-up appointment was not significantly associated with subsequent emergency department visits or hospital readmissions. ${ }^{9} \mathrm{~A}$ study from Texas showed that in a primarily commercially insured population, physicians had difficulty scheduling newborns for follow-up appointments according to AAP recommendations, citing provider knowledge and behavior as barriers to adherence. ${ }^{10}$

In this article, we describe a multipractice quality improvement learning collaborative (QILC) focused on improving timely newborn follow-up appointments. The QILC was launched after an AAP chapter meeting at which several pediatricians discussed common challenges to getting newborns seen promptly in the pediatric medical home. This learning collaborative brought together newborn hospitalists and outpatient pediatricians to discuss barriers and develop solutions, with the goal of improving processes across all sites. Timely newborn follow-up is defined in this project as an appointment in the pediatric medical home on any of the three calendar days following discharge from the well newborn nursery. Outpatient practices aimed to have at least $50 \%$ and $75 \%$ of newborns scheduled appropriately and $40 \%$ and $60 \%$ of newborns seen appropriately by 
three and six months into the project, respectively, with sustainment of progress at nine months. Inpatient hospitalist practices aimed to have at least $50 \%$ and $75 \%$ of newborns scheduled appropriately by three and six months into the project, respectively, with sustainment of progress at nine months.

\section{METHODS}

To improve timely newborn follow-up, a nine-month multipractice QILC was formed through a preexisting physician quality network. The Institute for Healthcare Improvement's Breakthrough Series was used as a model for the learning collaborative. ${ }^{11}$ The Indiana University Institutional Review Board determined that formal review was not required for this quality improvement project with no identifiable patient data being collected or stored.

Participating physicians were recruited through e-mail advertisement and registered through a website link. Physicians enrolled their practice in the QILC as either an outpatient, clinic-based practice or as an inpatient, hospital-based practice. Any physician who cares for newborns in either a pediatric clinic or a well newborn nursery was eligible to participate. Multiple physicians in a single practice were able to participate. Participating physicians were asked to champion the project within a multidisciplinary team to drive change within their practice setting. Practices were not targeted for recruitment or enrollment based on known gaps in newborn scheduling procedures.

The QILC was led by a pediatrician (project leader) with the support of a project coordinator who managed administrative tasks and analyzed data. Due to the distance between practice sites, the QILC was entirely virtual. Monthly learning collaborative webinars were led by the project leader and featured teaching on a quality improvement topic based on IHI's Model for Improvement ${ }^{11}$ ( Table 1 ). During each webinar, there was topic-based education on the importance of timely newborn follow-up. Reasons why newborns would need to be seen more promptly than three days after discharge (for example, a high-risk bilirubin value) were reviewed. In addition, webinars included presentation of individual practice and aggregate data through run charts and discussion of practices' Plan-Do-Study-Act (PDSA) cycles. Finally, practices discussed their successes and challenges in the project. The QILC orientation and baseline data collection occurred in September 2016. The learning collaborative and interventions occurred from October 2016 through July 2017, with the last session serving as a conclusion to the project. Pediatricians who submitted all required project data and attended at least six out of nine learning collaborative calls were eligible to receive American Board of Pediatrics (ABP) Maintenance of Certification Part 4 (MOC 4) credit sponsored by the Indiana University School of Medicine. 
Table 1

Learning Collaborative Quality Improvement Discussion Topics

\begin{tabular}{|c|c|}
\hline $\begin{array}{l}\text { Learning Collaborative } \\
\text { Session }\end{array}$ & $\begin{array}{c}\text { Quality Improvement Discussion } \\
\text { Topic }\end{array}$ \\
\hline 1 & $\begin{array}{l}\text { Forming a team and developing a } \\
\text { SMART aim }\end{array}$ \\
\hline 2 & $\begin{array}{l}\text { Developing changes and using } \\
\text { process maps }\end{array}$ \\
\hline 3 & Barriers to change in medicine \\
\hline 4 & Testing changes \\
\hline 5 & Using run charts \\
\hline 6 & $\begin{array}{l}\text { Sustaining change and using vi- } \\
\text { sual management }\end{array}$ \\
\hline 7 & Change management \\
\hline 8 & Spreading change \\
\hline 9 & Leading change \\
\hline
\end{tabular}

Learning collaborative participants collected data via monthly chart review. The participants collected data on all eligible newborns in their practice, up to 40 newborns per month, identifying eligible patients through review of daily schedules. Each practice received a survey link to enter data for each phase of the project. Each month was a different data collection phase. Data from each practice were submitted at baseline and in nine subsequent monthly phases throughout the learning collaborative. Data were submitted and managed using the secure, Web-based application REDCap (Research Electronic Data Capture), hosted at Indiana University, which provides an interface for validated data entry. 12 Run charts were created with Microsoft Excel (Microsoft Corp., Redmond, Washington) every month. Three-, six-, and nine-month aims were set prior to initiation of the QILC by the project leader based on known variability in local newborn follow-up appointment scheduling. Control charts were generated at the conclusion of the QILC through QI Macros for Excel (KnowWare International Inc., Denver) to assess the improvement in scheduling procedures from baseline.

Eligibility criteria were set by project leadership prior to the project launch, and the criteria were reviewed during the introductory webinar to guide data collection at practice sites. For outpatient practices, newborns were eligible for inclusion if they had been discharged from the well newborn nursery, were presenting for their first newborn appointment, and were younger than 1 month of age. Newborns were excluded if they were discharged 
from the neonatal ICU (NICU) or presented for their first appointment at older than 1 month of age. Newborns were excluded from inpatient data collection if they were discharged from the NICU.

As noted, timely newborn follow-up was defined as a newborn being scheduled for his or her first appointment within three calendar days of discharge home from the newborn nursery. The newborn could be scheduled with any pediatric provider within the child's medical home and did not have to be scheduled with his or her own assigned primary care physician. Outpatient practices collected data on the number of newborns scheduled for a timely newborn follow-up appointment and the number of newborns who completed that visit. For outpatient practices, the numerator was the number of newborns scheduled or seen appropriately, while the denominator was the total number of newborns presenting for the first newborn appointment during each phase of monthly data collection. Inpatient practices collected data on the number of newborns who were scheduled for a timely newborn follow-up appointment. The numerator for inpatient practices was the number of newborns scheduled appropriately, while the denominator was the total number of newborns discharged from the well newborn nursery during each phase of monthly data collection. Because newborn hospitalists sent patients to pediatric offices within several health care organizations with different electronic medical records, the inpatient practices were unable to track the completion of scheduled newborn followup appointments.

\section{RESULTS}

Twenty-four physicians from nine outpatient practices and three inpatient practices participated in the QILC. One practice submitted data as both an outpatient and inpatient practice. An additional outpatient practice enrolled in the QILC but dropped out after phase 4 , citing time constraints of the physician champion. All other sites submitted data during each phase of the project. The outpatient practices were located throughout the state of Indiana and varied in size from a small, solo-practitioner office to large, academic federally qualified health centers. Participating inpatient practices ranged from highvolume newborn hospitalist groups with teaching services to a community maternity center.

Baseline data revealed that enrolled outpatient practices had a wide variety of newborn scheduling procedures that ranged from $43 \%$ to $94 \%$ of newborns scheduled for their first newborn follow-up visit within three days of hospital discharge ( Table 2 ). Similarly, $43 \%$ to $93 \%$ of newborns were seen in their primary medical home appropriately at baseline ( Table 3 ). Inpatient practices also varied in their newborn scheduling procedures at baseline, with $73 \%$ to $100 \%$ of newborns scheduled for their first newborn appointment appropriately ( Table 2 ). All inpatient practices and seven out of eight outpatient practices that completed the QILC met the goal of $75 \%$ of newborns scheduled for their first newborn appointment appropriately ( Table 2 ). All outpatient practices met the goal of $60 \%$ of newborns seen by their primary care providers within three days of hospital discharge ( Table 3 ). Most outpatient practices were able to sustain their improvements, 
with only one practice finishing the learning collaborative at a lower point than its baseline data.

\section{Table 2}

Percentage of Newborns with Timely Newborn Follow-Up_Patient Scheduled

\begin{tabular}{|lcc|}
\hline \multicolumn{3}{|l|}{$\begin{array}{l}\text { Table 2. Percentage of Newborns with Timely New- } \\
\text { born Follow-Up-Patient Scheduled }\end{array}$} \\
\hline & Baseline \% & Phase 9\% \\
\hline Outpatient Practice 1 & 83 & 100 \\
Outpatient Practice 2 & 92 & 100 \\
Outpatient Practice 3 & 93 & 100 \\
Outpatient Practice 4 & 43 & Did not complete \\
Outpatient Practice 5 & 63 & 100 \\
Outpatient Practice 6 & 94 & 100 \\
Outpatient Practice 7 & 71 & 60 \\
Outpatient Practice 8 & 72 & 100 \\
\hline Outpatient Practice 9 & 91 & 100 \\
Inpatient Practice 1 & 100 & 100 \\
\hline Inpatient Practice 2 & 73 & 93 \\
Inpatient Practice 3 & 95 & 100 \\
\hline
\end{tabular}

\section{Table 3}

Percentage of Newborns with Timely Newborn Follow-Up_Patient See

\begin{tabular}{|lcc|}
\hline \multicolumn{3}{|l|}{$\begin{array}{l}\text { Table 3. Percentage of Newborns with Timely New- } \\
\text { born Follow-Up-Patient Seen }\end{array}$} \\
\hline & Baseline \% & Phase 9\% \\
\hline Outpatient Practice 1 & 83 & 100 \\
Outpatient Practice 2 & 90 & 96 \\
Outpatient Practice 3 & 86 & 100 \\
Outpatient Practice 4 & 43 & Did not complete \\
Outpatient Practice 5 & 63 & 83 \\
Outpatient Practice 6 & 91 & 67 \\
Outpatient Practice 7 & 71 & 100 \\
Outpatient Practice 8 & 72 & 100 \\
\hline Outpatient Practice 9 & 91 & \\
\hline
\end{tabular}

Aggregate data from the practices showed continual improvement in all measured newborn scheduling metrics throughout the nine-month learning collaborative except for 
phase 3. Phase 3 occurred during December, when many pediatric offices were closed for several days over the holidays, leading to delays in appointment scheduling. Improvement was sustained over the last three months of the learning collaborative (Figures 1-3). By the end of the learning collaborative, outpatient practices were scheduling newborns for their first appointment appropriately $92 \%$ of the time, up from an aggregate baseline of $78 \%$ ( Figure 1). Likewise, newborns were seen for their first newborn appointment appropriately $89 \%$ of the time, up from an aggregate baseline of $76 \%$ ( Figure 2 ). Inpatient, hospital-based practices scheduled newborns for their first outpatient appointment within three days of discharge $99 \%$ of the time, up from a baseline of $89 \%$ ( Figure 3 ). Both outpatient metrics showed a statistical change with a center line shift (Figures 1 and 2 ). Inpatient scheduling processes showed a trend toward improvement but no statistical change ( Figure 3 ).

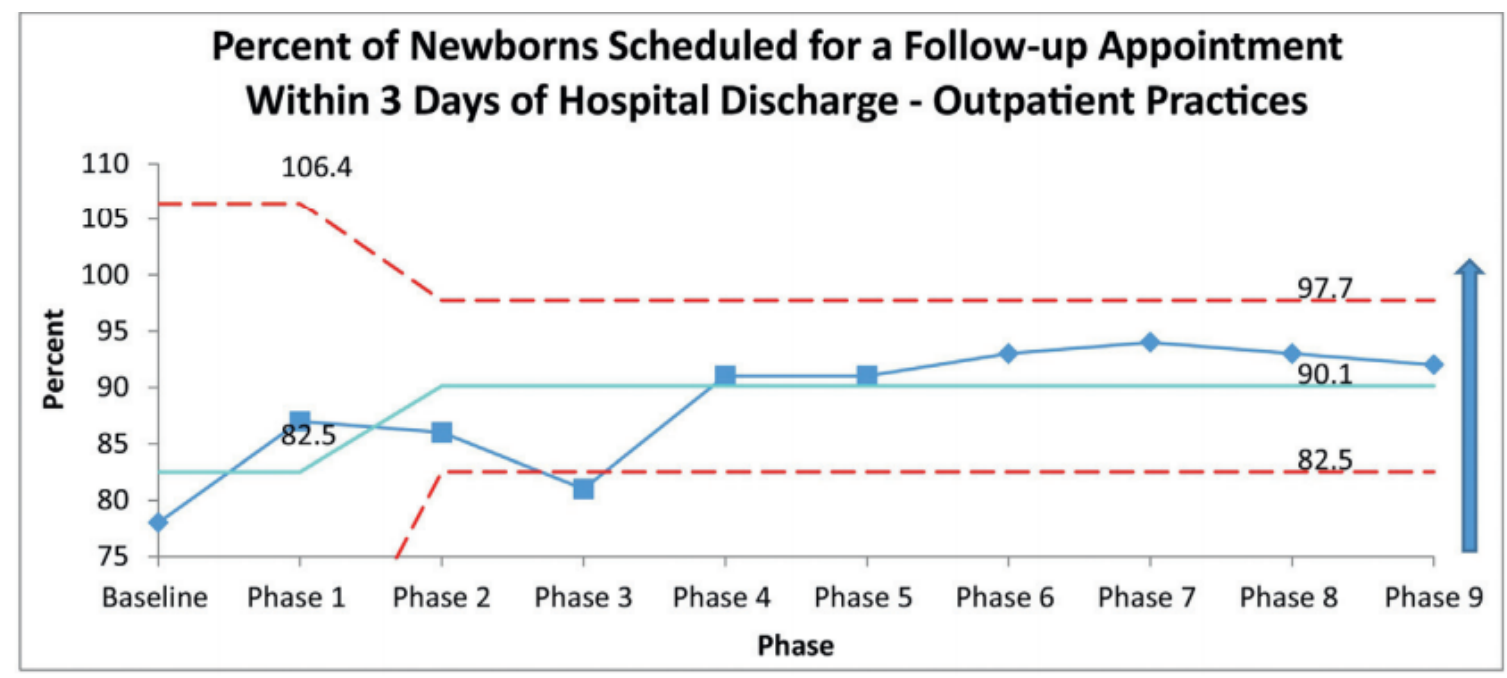

\section{Figure 1}

In this chart, the blue line shows the percentage of newborns scheduled for a timely (within three days of hospital discharge) newborn follow-up appointment in all outpatient practices (aggregate data)

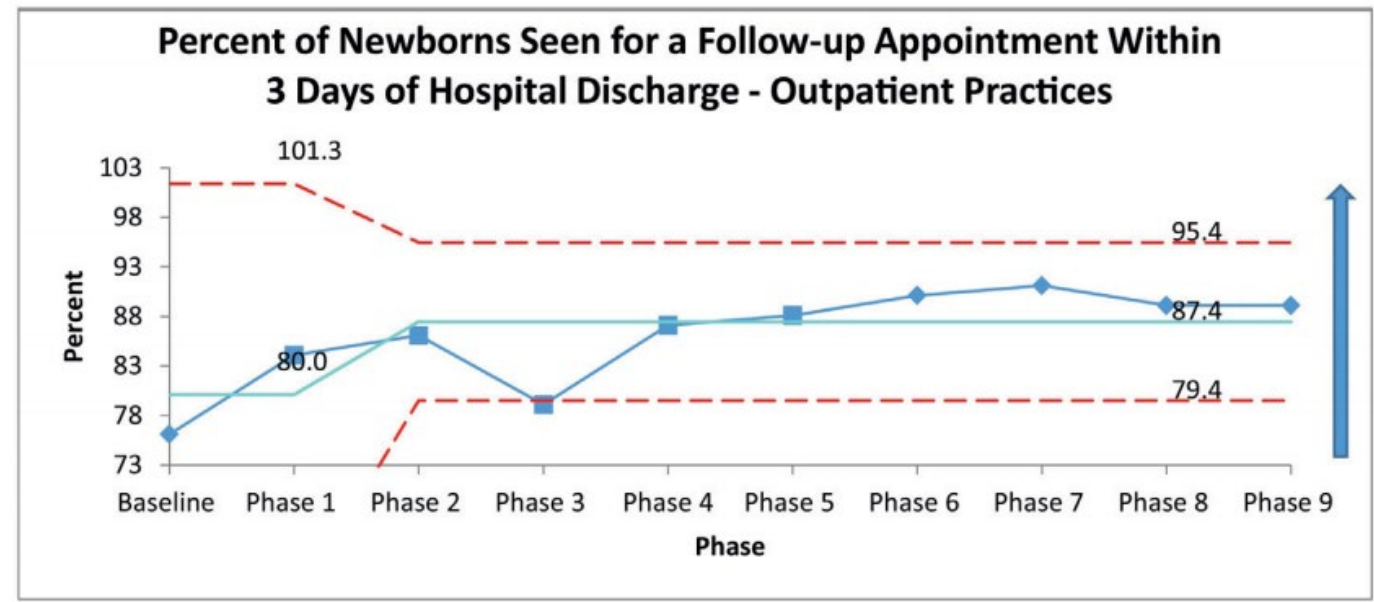




\section{Figure 2}

In this chart, the blue line shows the percentage of newborns seen for a timely (within three days of hospital discharge) newborn follow-up appointment in all outpatient practices (aggregate data).

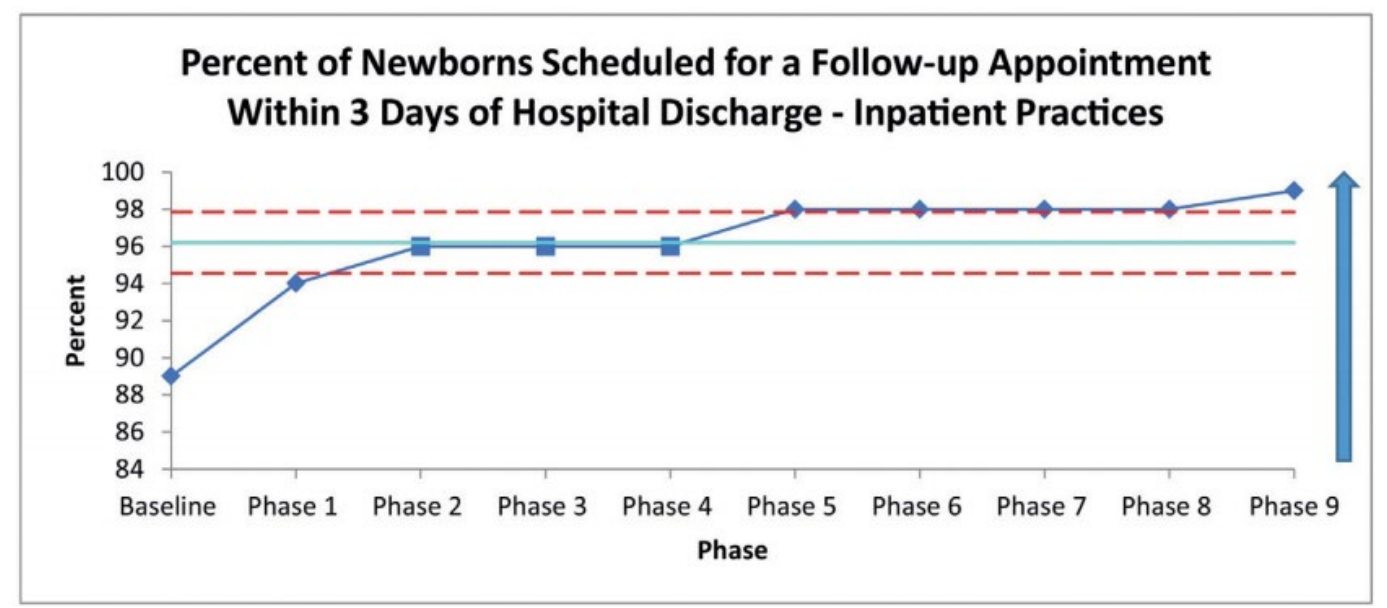

\section{Figure 3}

In this chart, the blue line shows the percentage of newborns scheduled for a timely (within three days of hospital discharge) newborn follow-up appointment in all inpatient practices (aggregate data).

\section{DISCUSSION}

Each practice created a multidisciplinary improvement team and implemented changes to improve newborn scheduling based on its own identified individual needs. Multiple PDSA cycles were undertaken by each practice. All offices educated physicians, nurses, and front office staff on the importance of timely newborn appointment scheduling. Expectations were set with front office staff that newborns had to be scheduled within three days of discharge. For newborn appointment "no shows," processes were put in place to contact families and reschedule appointments within the following 24 hours whenever possible. Some offices created open newborn slots in their schedule every day that could be filled with sick visits if not needed for newborn follow-up. Inpatient units developed a process to schedule appointments for newborns discharged over the weekend, which had been identified as an early barrier. An inpatient hospitalist group and outpatient practice worked together to develop a process for screening families for transportation barriers at the time of scheduling and helped the family access services, such as a Medicaid cab, if needed. Standard work and visual management were created to standardize the new scheduling process and track progress. Successful and unsuccessful PDSA cycles were shared during monthly QILC webinars. Practices modified each other's successful changes to try in their own practice site. 
The biggest barrier faced by project participants was a lack of knowledge about the AAP recommendations for timely newborn follow-up. Championing physicians acknowledged that fellow physicians, nurses, office staff, and patients were frequently unaware of the need for close newborn follow-up after hospital discharge. Outpatient practices also noted that families were not always instructed to schedule their first newborn appointment within two to three days by the newborn nursery staff, causing the office to not receive a phone call to set up the initial appointment until the baby was older than five to seven days. In addition, physicians struggled with scheduling timely appointments for newborns discharged home on Thursdays, consistent with experience reported by other groups. ${ }^{8}$ Improved and consistent education about the importance of early newborn follow-up visits, both in the medical community and the general public, may improve compliance with recommendations.

The intent of this QILC was for improved timely newborn scheduling processes to be incorporated into the sites' permanent workflow. Although sustainment of improvement was evaluated over the final three months of the QILC, metrics are no longer being formally tracked and the authors have not resurveyed practices to ensure that newborns are still being scheduled within three days of hospital discharge. A weakness of this project is that project aims were set by the leader prior to enrollment of the sites. Baseline data revealed that most practices were exceeding the three-month aim of $50 \%$ of newborns scheduled appropriately, which likely set the project aims too low. In addition, participation in the learning collaborative was voluntary. Motivated, engaged participants may skew data toward showing improvement. This project also lacked an independent control group to compare outcomes. Finally, no clinical outcomes were tracked during the QILC. The authors are unable to comment on whether improved adherence to the AAP's newborn scheduling recommendations decreased morbidity for the patient population.

A valuable lesson learned from this QILC was the importance of tying quality improvement work to MOC 4 credit for physicians. When surveyed at the end of the learning collaborative, participating pediatricians cited the availability of MOC 4 credit from the $\mathrm{ABP}$ as a major driver for participation. The requirement to attend two thirds of the webinars and to submit data for all phases to receive MOC 4 credit likely led to increased participation and engagement. The single practice that dropped out of the QILC was a family medicine practice, and the project team had been unable to secure MOC 4 credit for participating family medicine physicians.

\section{CONCLUSION}

This project shows that QILCs can be successful in driving improvement across multiple practices. This mirrors the results of several other recent studies showing success of multisite learning collaboratives improving implementation of evidence-based practices in the newborn nursery 1314 and outpatient pediatric clinic ${ }^{15}$ using quality improvement methodology. This QILC was unique in that outpatient and inpatient practices collaborated to solve a problem around transition of care for newborns. Although inpatient practices were more adherent to the AAP's newborn follow-up recommendations at 
baseline, their insight was invaluable to discussions on barriers and solutions to newborn appointment scheduling.

\section{REFERENCES}

1. Young P.C., Korgenski K., Buchi K.F.: Early readmission of newborns in a large health care system. Pediatrics 2013; 131: pp. e1538-e1544.

2. Hagan J.F.Shaw J.S.Duncan P.M.Bright Futures: Guidelines for Health Supervision of Infants, Children, and Adolescents.2017.American Academy of PediatricsElk Grove Village, IL

3. Meek J.Y., Hatcher A.J.: The breastfeeding-friendly pediatric office practice. Pediatrics 2017; 139

4. American Academy of Pediatrics Subcommittee on Hyperbilirubinemia : Management of hyperbilirubinemia in the newborn infant 35 or more weeks of gestation. Pediatrics 2004; 114: pp. 297-316.

5. Benitz W.E.: Hospital stay for healthy term newborn infants. Pediatrics 2015; 135: pp. 948-953.

6. Shakib J., et. al.: Timing of initial well-child visit and readmissions of newborns. Pediatrics 2015; 135: pp. 469-474.

7. Meara E., et. al.: Impact of early newborn discharge legislation and early follow-up visits on infant outcomes in a state Medicaid population. Pediatrics 2004; 113: pp. 1619-1627.

8. O'Donnell H.C., et. al.: Factors associated with timing of first outpatient visit after newborn hospital discharge. Acad Pediatr 2014; 14: pp. 77-83.

9. O'Donnell H.C., et. al.: Impact of newborn follow-up visit timing on subsequent ED visits and hospital readmissions: an instrumental variable analysis. Acad Pediatr 2014; 14 : pp. 84-91.

10.Profit J., et. al.: Delayed pediatric office follow-up of newborns after birth hospitalization. Pediatrics 2009; 124: pp. 548-554.

11. Institute for Healthcare Improvement : The Breakthrough Series: IHl's Collaborative Model for Achieving Breakthrough Improvement. IHI Innovation Series white paper. http://www.ihi.org/resources/Pages/IHIWhitePapers/TheBreakthroughSeriesIHIsCollab orativeModelforAchievingBreakthroughlmprovement.aspx 
12. Harris P.A., et. al.: Research Electronic Data Capture (REDCap)—a metadata-driven methodology and workflow process for providing translational research informatics support. J Biomed Inform 2009; 42: pp. 377-381.

13. Kellams A., et. al.: TodaysBaby quality improvement: safe sleep teaching and role modeling in 8 US maternity units. Pediatrics 2017; 140

14.Walsh M.C., et. al.: Ohio Perinatal Quality Collaborative improves care of neonatal narcotic abstinence syndrome. Pediatrics 2018; 141

15. Beers L.S., et. al.: Mental health screening quality improvement learning collaborative in pediatric primary care. Pediatrics 2017; 140 